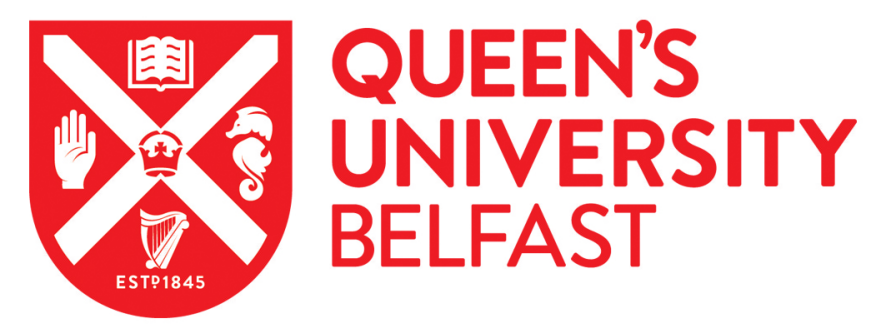

\title{
123I-MIBG scintigraphy utility and cut-off value in a clinically representative dementia cohort
}

Kane, J. P. M., Roberts, G., Petrides, G. S., Lloyd, J. J., O'Brien, J. T., \& Thomas, A. J. (2019). 123I-MIBG scintigraphy utility and cut-off value in a clinically representative dementia cohort. Parkinsonism and Related Disorders. https://doi.org/10.1016/j.parkreldis.2019.01.024

Published in:

Parkinsonism and Related Disorders

Document Version:

Peer reviewed version

Queen's University Belfast - Research Portal:

Link to publication record in Queen's University Belfast Research Portal

Publisher rights

Copyright 2019 Elsevier.

This manuscript is distributed under a Creative Commons Attribution-NonCommercial-NoDerivs License

(https://creativecommons.org/licenses/by-nc-nd/4.0/), which permits distribution and reproduction for non-commercial purposes, provided the author and source are cited.

\section{General rights}

Copyright for the publications made accessible via the Queen's University Belfast Research Portal is retained by the author(s) and / or other copyright owners and it is a condition of accessing these publications that users recognise and abide by the legal requirements associated with these rights.

Take down policy

The Research Portal is Queen's institutional repository that provides access to Queen's research output. Every effort has been made to ensure that content in the Research Portal does not infringe any person's rights, or applicable UK laws. If you discover content in the Research Portal that you believe breaches copyright or violates any law, please contact openaccess@qub.ac.uk. 


\title{
${ }^{123}$ I-MIBG scintigraphy utility and cut-off value in a clinically representative dementia cohort
}

\author{
JPM Kane ${ }^{1 *}$, G Roberts ${ }^{1,2}$, GS Petrides ${ }^{2}$, JJ Lloyd ${ }^{1,2}$, JT O'Brien ${ }^{1,3}$, AJ Thomas ${ }^{1}$ \\ Corresponding author \\ 1 Institute of Neuroscience, Newcastle University \\ Biomedical Research Building \\ Campus for Ageing and Vitality \\ Newcastle University \\ Newcastle upon Tyne \\ NE4 5PL \\ United Kingdom \\ $2 \quad$ Nuclear Medicine department \\ Royal Victoria Infirmary \\ Newcastle upon Tyne NHS Foundation Hospitals Trust \\ Queen Victoria Road \\ Newcastle upon Tyne \\ NE1 4LP \\ United Kingdom
}

3 Department of Psychiatry, University of Cambridge

Box 189, Level E4 Cambridge Biomedical Campus

Cambridge

CB2 OSP

United Kingdom

Dr Joseph PM Kane

Ms Gemma Roberts

Dr George S Petrides

Dr James J Lloyd

Professor JT O'Brien

Professor AJ Thomas j.kane2@newcastle.ac.uk gemma.roberts@newcastle.ac.uk george.petrides@nuth.nhs.uk j.j.lloyd@newcastle.ac.uk john.obrien@medschl.cam.ac.uk alan.thomas@newcastle.ac.uk 


\section{CORRESPONDING AUTHOR}

\section{Joseph PM Kane}

Institute of Neuroscience

Biomedical Research Building

Campus for Ageing and Vitality

Newcastle University

Newcastle upon Tyne

NE4 5PL

Tel 01912081318

Email j.kane2@newcastle.ac.uk

Word count* 2990

Abstract word count* $\quad 248$

Tables 2

Figures 2

References $\quad 30$

(*Excluding headings, figures, tables and acknowledgements) 


\section{ABSTRACT}

\section{Objective}

To determine the utility of ${ }^{123}$ I-metaiodobenzylguanidine cardiac scintigraphy (MIBG), and optimum heart: mediastinum ratio (HMR) for differentiating dementia with Lewy bodies (DLB) from Alzheimer's disease (AD) in a clinically representative population, comparing

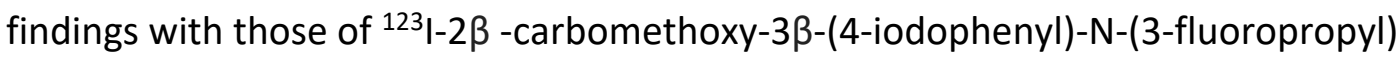
nortropane (FP-CIT) SPECT.

\section{Methods}

We recruited subjects with probable DLB $(n=17)$ and probable $A D(n=16)$ from clinical services. Each participant underwent clinical examination, cardiac MIBG scintigraphy and FP-CIT SPECT. Diagnosis was made on the basis of clinical symptoms using validated criteria. Cardiac MIBG uptake was measured by the planar HMR, blind to clinical diagnosis, with values below a cut-off taken from a previous study ( $<2.2$ at four hours) defining scans as abnormal. FP-CIT scans were blindly rated according to a visual rating scale.

\section{Results}

MIBG had a sensitivity, specificity and overall accuracy of $71 \%, 81 \%$ and $76 \%$ for distinguishing DLB from AD. FP-CIT demonstrated a sensitivity, specificity and accuracy of $82 \%, 88 \%$ and $85 \%$. Using a lower HMR cut-off to distinguish between abnormal and normal MIBG scans improved the accuracy of MIBG, raising specificity $(100 \%)$ and overall accuracy (85\%) without compromising sensitivity (71\%). Neither prescription of potentially interfering medications, nor a history of myocardial infarction (MI), had a significant effect on HMR. 


\section{Conclusion}

We found that MIBG did not demonstrate superior sensitivity and overall accuracy to FP-CIT. HMR cut-off influences biomarker utility, and clinical and Caucasian populations may require a lower cut-off than those reported elsewhere. Future MIBG studies should include clinically representative cohorts as neither medications nor previous MI appear to influence HMR. 


\section{BACKGROUND}

Characterised by a tetrad of visual hallucinations, spontaneous parkinsonism, cognitive fluctuations and REM sleep behaviour disorder[1], dementia with Lewy bodies (DLB) comprises $5-10 \%$ of dementia cohorts[2,3]. DLB recognition has important implications for clinical management and carer wellbeing, but initial misdiagnosis outside the specialist setting is common[4,5].

${ }^{123}$ I-metaiodobenzylguanidine (MIBG) scintigraphy was recently included as an indicative biomarker in the fourth DLB consensus criteria[1]. Cardiac sympathetic denervation is among the many manifestations of extracranial $\alpha$-synuclein pathology[6] and MIBG, an analogue of noradrenaline, allows in vivo measurement of cardiac uptake relative to a mediastinal reference uptake as a heart: mediastinum ratio (HMR). This HMR is suggestive of DLB when it falls below a predetermined cut-off, but no consensus figure for this cut-off has yet been agreed upon, and previous studies of MIBG utility have used a range of different values[7-10].

Single centre studies have reported excellent rates of sensitivity (94-100\%) and specificity (87-100\%) for distinguishing DLB from Alzheimer's disease (AD)[7-9]. A recent multicentre study, representing the largest sample size in the field to date, found lower sensitivity (69\%) and specificity (89\%)[10], but these increased to $77 \%$ and $97 \%$ upon diagnostic reappraisal after three years[11].

The lower diagnostic accuracy, and higher proposed cut-off in the multi-site study indicates that some factors may limit generalisability of MIBG to typical clinical populations, including 
the exclusion of patients with comorbidities like diabetes $[9,12,13]$ and myocardial infarction (MI) $[12,13]$ from many studies. This is particularly relevant given the higher prevalence of such conditions in Western countries than in Japan[14], where the majority of MIBG research has taken place.

Previous studies have also excluded patients prescribed medications suspected of interfering with MIBG uptake[15], or asked subjects to temporarily withdraw them[16], but little evidence exists regarding the impact of individual medications[17]. This would be important to know if MIBG is to be used as a standard clinical diagnostic test.

${ }^{123}$ I-FP-CIT SPECT (FP-CIT), also included as an indicative marker in consensus criteria[1], identifies nigrostriatal degeneration often seen in DLB by visualising impaired radioisotope uptake at the striatal dopamine reuptake transporter site. More widely used than MIBG in clinical settings, FP-CIT is supported by a more substantial evidence base that includes autopsy validation[18]. A specificity of $90 \%$ has been cited for FP-CIT, but a reported sensitivity of $77 \%$ presents the likelihood of false negative findings in many cases[19]. Abnormal FP-CIT findings can be seen in multisystem atrophy, progressive supranuclear palsy and frontotemporal dementia, which could lead to false positive DLB diagnoses[20,21]. In contrast, MIBG has demonstrated the capability to distinguish DLB from these other disorders $[9,12]$.

This study aimed to investigate the utility of MIBG cardiac scintigraphy in differentiating DLB from Alzheimer's disease (AD) in a clinically representative cohort and to determine the most appropriate HMR cut-off for this population. It also aimed to compare concurrent 
findings of MIBG and FP-CIT to help inform clinicians about the choice and order of preference of these two biomarkers.

\section{METHOD}

\section{Recruitment}

Subjects over 60 years old with probable AD or probable DLB were recruited through Psychiatry of Old Age, geriatric medicine and neurology clinics, or research volunteer registers in North-East England between September 2015 and June 2017. Written informed consent was provided by patients, or by a nominated consultee when participants did not demonstrate capacity to consent for themselves.

Exclusion criteria were limited to factors demonstrated to interfere with MIBG uptake, such as symptomatic cardiac failure[22]. A history of MI only excluded patients where it had occurred within one year of recruitment, as post-infarction reinnervation has been demonstrated[22].

Patients prescribed labetalol were excluded; labetalol, unlike other beta-receptor antagonists without alpha activity, is recognised as inhibiting cardiac MIBG uptake[17]. Those prescribed tricyclic antidepressants were also excluded for the same reason[17]. Participants prescribed all other agents were included.

Each subject underwent a detailed clinical assessment, including neurological examination and neuropsychological testing by a specialist medical practitioner (JK). As in our previous studies[18,19], diagnosis was reviewed independently by two diagnosticians (JK, AT) blinded 
to patient identity and scan results, who applied the fourth consensus criteria for DLB[1], and NIA-AA criteria for AD[23]. Where disagreement arose between raters, a third diagnostician (JOB) provided a deciding opinion.

Image acquisition

For both FP-CIT and MIBG, patients were administered their medication as routinely prescribed. Each received $170 \mathrm{mg}$ of oral potassium iodate one hour prior to intravenous delivery of $111 \mathrm{MBq}$ of ${ }^{123}$ I-MIBG or $185 \mathrm{MBq}$ of ${ }^{123}$ I-FP-CIT. The same dose of potassium iodate was administered 12 hours after MIBG injection. Except in cases where patients had received FP-CIT scan as part of their routine clinical care, MIBG scans were conducted first, and FP-CIT scans second.

\section{MIBG cardiac scintigraphy}

MIBG and FP-CIT scans were conducted using one of two dual-headed gamma cameras (Siemens Symbia T Series or Siemens Symbia Intevo). For MIBG, these were fitted with medium energy general purpose collimators. Cameras were calibrated using a planar cardiac MIBG phantom, adopting the method employed by Nakajima and colleagues[24]. This allowed conversion of the 2.1 cut-off reported by Yoshita et al. for both early and late images[10] to a 2.2 cut-off adopted for use in this study. Early and late planar images were obtained at 20 and 240 minutes following MIBG injection, acquired over 10 minutes, and stored in a $128 \times 128$ matrix.

One rater (GR) used a methodology consistent with previous studies to assess blinded planar MIBG images[10,16]. A myocardial ("heart") region of interest (ROI) 
was circumscribed using a freehand tool (Figure 1) and a mediastinal ROI of area 48 pixels was placed on the midline of the upper chest. HMR values provided by a second practitioner (JK) were used to determine interrater reliability, but not included in analysis of HMR in relation to diagnosis, medication use and history of MI.

\section{FP-CIT SPECT}

All subjects underwent SPECT within 3-6 hours of ${ }^{123} \mathrm{FP}-\mathrm{CIT}$ injection. Cameras were fitted with low-energy high resolution collimators and acquired 120 views in a circular orbit. Images were stored in a $128 \times 128$ matrix.

Transverse images were reconstructed using a ramp-filtered back projection with a Butterworth filter (cut-off 0.3 cycles/cm; order 10), without correcting for gamma ray attenuation. For visual analysis, 16 slices of $1.95 \mathrm{~mm}$ thickness were displayed using the " $G E$ " colour scale. Images were assessed by five independent raters blinded to patient identity and clinical information: one consultant medical radiologist, two consultant medical physicists experienced in nuclear medicine reporting, and two certified old age psychiatrists (GP, GR, JL, AT, JK). Each completed a training exercise prior to assessing study images, comprising familiarisation with the visual rating scale using independent FP-CIT images ranging from normal to markedly abnormal.

Scans were rated as normal or abnormal, and given a visual rating; 0 (normal uptake in all regions), 1 (asymmetric activity with one putamen showing reduced uptake), 2 (absent activity in the putamen of both hemispheres), or 3 (absent activity in the putamen of both 
hemispheres and greatly reduced in one or both caudate nuclei)[25]. Consensus ratings for normal/abnormal scans, and visual ratings, were agreed upon, as previously reported[19].

\section{Statistical analysis}

Analysis was performed using SPSS 24.0. Continuous and categorical variables were analysed using Student's $t$ test for independent samples and $\chi^{2}$ test respectively. MannWhitney test was used for non-parametric data. We calculated sensitivity, specificity, and diagnostic accuracy using 2×2 tables and confidence intervals using Microsoft Excel 2016. 


\section{RESULTS}

Of 125 patients approached to take part in the study, 43 consented to participate. Seven subjects did not undergo MIBG or FP-CIT due to death before scanning could be arranged, ill health or withdrawal. Three subjects were excluded from analysis; two on the basis of an expert panel diagnosis other than probable DLB or $A D$ and one due to a deviation in protocol. Seventeen patients with probable DLB and 16 with probable AD were therefore included in analysis.

Groups were comparable in age, gender, and cognitive profile (Table 1). As expected, DLB cases had higher scores on the Unified Parkinson's Disease Rating Scale (UPDRS), Dementia Cognitive Fluctuations Scale (DCFS) and the hallucinations subscale of the Neuropsychiatric Inventory (NPI). The NPI subscale records the frequency and severity of all hallucinations but could be used in this study as a measure of visual hallucinations, as no subject described hallucinations in any other modalities.

Higher levels of functional impairment and neuropsychiatric symptoms were found in patients with DLB. Scans were conducted a within a mean of 76 (SD \pm 101 ; range 7- 401) days of each other. 
We observed abnormal HMRs $<2.2$ in twelve patients with DLB $(12 / 17)$ and three with AD (3/16) (Figure 2), corresponding to a sensitivity, specificity and overall accuracy of $71 \%$ (95\% Cl 44-90\%), 81\% (95\% Cl 54-96\%), and 76\% (95\% Cl 55-87\%). Eleven patients with DLB (11/17) and four with AD (4/17) had abnormal early HMRs, corresponding to a sensitivity, specificity and accuracy of $65 \%$ (38-86\%), 75\% (47-93\%) and 70\% (51-84\%). The two raters' normal/ abnormal late MIBG result agreed on 97\% (32/33) of cases (Cohen's kappa $=0.94)$. Overall, MIBG uptake was significantly lower in DLB for both late and early HMRs (late HMR: probable DLB $(1.65 \pm 0.71)$, probable AD $(2.39 \pm 0.38) ; U=55.0, p<0.01$; early HMR: DLB $(1.82$ $\pm 0.62), A D(2.41 \pm 0.41) ; U=56.5, p<0.01)$.

FP-CIT FP-CIT sensitivity, specificity and accuracy were 82\% (95\% Cl 65-93\%), 88\% (95\% Cl 6499\%) and $85 \%$ (95\% Cl 68-95\%) respectively. Both MIBG and FP-CIT were positive in 65\% of DLB patients (11/17; $95 \% \mathrm{Cl} 41-83 \%)$ and were both negative in $69 \%$ of AD patients $(11 / 16$; $95 \% \mathrm{Cl} 44-86 \%)$. Six cases $(6 / 16 ; 38 \%)$ demonstrated unilateral impaired putaminal uptake and five (31\%) bilateral impaired putaminal uptake. Five cases (31\%) showed impaired uptake in both putamina and at least one caudate nucleus.

\section{Sensitivity analysis}

The time between scans was greater than six months in five cases (15\%; 5/33). When these were removed from analysis, the sensitivity, specificity and accuracy of were $67 \%(95 \% \mathrm{Cl}$ 35-90\%), 81\% (95\% Cl 54-96\%), and 75\% (95\% Cl 55-89\%) for MIBG and 83\% (95\% Cl 5298\%), 75\% (95\% Cl 48-93\%) and 79\% (95\% Cl 59-92\%) for FP-CIT. 
ROC analysis for MIBG cut-off

Since four patients with $A D$ and normal FP-CIT imaging had $\mathrm{HMR}<2.2$, we conducted a receiver operator curve $(\mathrm{ROC})$ analysis, which identified an area under the curve of 0.80 (95\% $\mathrm{Cl} 0.63-0.96)$ and an optimum late HMR cut-off of 1.6. Without compromising sensitivity (71\%; 95\% Cl 44-90), this post hoc cut-off would confer superior specificity (100\%; $95 \% \mathrm{Cl} 79-100)$ and overall accuracy (85\%; $95 \% \mathrm{Cl} 68-95)$ than the a priori cut-off.

\section{Potentially interacting medications}

Twenty-three medications identified as potentially interacting with MIBG uptake were prescribed to twelve patients with DLB $(12 / 17 ; 71 \%)$ and six with AD $(6 / 16 ; 38 \%)$ (Table 2). No significant difference in late HMR was identified between patients prescribed these agents $(1.79 \pm 0.68)$ and those that were not $(2.27 \pm 0.60 ; U=89.0, p=0.10)$. No significant relationships between HMR and drug prescription were observed in either DLB (interacting medications ( $1.53 \pm 0.61)$, no interacting medications (1.94 \pm 0.93$) ; U=18.5, p=0.23$ ) or $A D$ groups (interacting medications $(2.30 \pm 0.52)$, no interacting medications $(2.43 \pm 0.29)$; $\mathrm{U}=30.0, p=0.80)$.

Since levodopa was only prescribed to patients with DLB, it is difficult to distinguish any drug effect from disease effect. We therefore repeated the analysis, excluding levodopa compounds, and found that late HMRs in the two groups were similar (prescribed potentially interacting medications $(1.92 \pm 0.70)$, no interacting medications $(2.06 \pm 0.68) ; \mathrm{U}$ $=126.0, \mathrm{p}=0.98)$ (Figure 2). Of patients prescribed interfering medications, we observed false positive MIBG results in two AD cases (2/6; 33\%), both taking amlodipine, and false negative results in three DLB cases, one taking amlodipine. We identified no significant 
relationship between amlodipine prescription and late HMR (prescribed amlodipine (1.93 \pm $0.65)$; not prescribed amlodipine $(2.03 \pm 0.70) ; U=90.0, p=0.93)$.

Myocardial infarction

Five subjects $(5 / 33 ; 15 \%)$, two with DLB $(2 / 17 ; 12 \%)$ and three with $A D(3 / 16 ; 19 \%)$ had a history of MI. None of the AD cases in question had an abnormal MIBG result. No significant difference in late HMR was observed between patients with a history of MI (2.26 \pm 0.71$)$ and those without $(1.96 \pm 0.68 ; U=51.5, p=0.36)$. 


\section{DISCUSSION}

We found that MIBG, at a pre-specified HMR cut-off of 2.2 , had a sensitivity, specificity and accuracy of $71 \%, 81 \%$ and $76 \%$. However, using a lower 1.6 cut-off to distinguish between abnormal and normal scans could improve the accuracy of MIBG (85\%), raising specificity (100\%) without compromising sensitivity (71\%). Our findings show a lower diagnostic accuracy than those seen in previous single-centre studies (sensitivity $>90 \%$, specificity $>87 \%)[8,9,12,13]$, but are more in keeping with the sensitivity and specificity of $69 \%$ and $89 \%$ reported by a multicentre study[10].

Our a priori cut-off was determined using phantom calibration with those used by Yoshita and colleagues[24] and assumed that MIBG uptake differences would be similar in our Caucasian population to those in Japan. This assumption does not seem to be accurate, and is further challenged by the higher cut-off proposed by the follow up of patients from Yoshita and colleagues' study[11]. Our post hoc HMR cut- off of 1.6 is lower than that reported elsewhere and might be explained the higher rates of obesity in the UK than in Japan, or the comparatively high age of our sample (DLB 77.4 $\pm 8.0 ; A D 76.6 \pm 6.8$ ) compared to the cohort described by Yoshita and colleagues (DLB 76.9 $\pm 5,4 ; A D 74.5 \pm 7.3$ ). Although some studies report comparable cut-off values[8], their methods used low energy collimators expected to produce lower HMRs[26]. Variation in appropriate cut-offs may exist in different populations and influence MIBG utility; determination of cut-offs that consider population-specific factors will be an important step in the integration of MIBG into routine clinical practice. 
We identified no significant relationship between late HMR and medications suspected to interfere with MIBG. Over half of the patients in our study $(18 / 33 ; 56 \%)$ were prescribed such medications, reflecting the high use of these drugs in clinical practice, and underlining the infeasibility in restricting MIBG use in such patients.

Despite the theoretical expectation that calcium channel blockade would suppress sympathetic activity, clinical studies investigating the role of amlodipine in MIBG have reported an increase or no significant effect on HMR[17]. Several factors, including dose, frequency, compliance, and pharmacokinetic variability could influence the relationship between any medication and HMR. Caution should be exercised in interpreting MIBG results in patients prescribed potentially interfering medications, but our data cast doubt on any suggestion that such factors should contraindicate MIBG.

We identified no significant relationship between HMR and MI. The United Kingdom (UK) prevalence of $\mathrm{Ml}$ is reported as $12.1 \%$ in men and $5.5 \%$ in women over 75 years old[27], a substantial proportion to consider excluding when appraising the clinical utility of MIBG. Several covariates, such as the time, location and extent of ischaemia are likely to be important factors in exploring the relationship between MI and MIBG uptake. Although our sample size is too small to dismiss the significance of such comorbidities on either MIBG utility or optimum cut-off, the lack of a significant relationship between HMR and MI status is encouraging.

Our observation that three patients with clinical DLB demonstrated no evidence of sympathetic cardiac denervation, as measured by MIBG, but did show striatal dopaminergic 
degeneration, as determined by FP-CIT, is important as it challenges the caudorostral hypothesis of Lewy body disease pathogenesis. Our data joins a growing body of evidence disputing this theory, and supports the suggestion that multiple patterns of pathogenesis may exist[28]. However, the presence of a normal HMR does not necessarily exclude the possibility of cardiac $\alpha$-synuclein deposition in DLB patients[29], and the correlation of neuropathological and MIBG data remains an important area for future research.

We found FP-CIT to have lower accuracy than that reported in a large multicentre study $[18,19]$. FP-CIT did, however, demonstrate marginally superior sensitivity and accuracy, with equivalent specificity, to that of MIBG in our cohort. Our findings may support using FP-CIT in preference to MIBG, but other factors may encourage clinical use of MIBG; early HMR findings (20 minutes post injection) were equivalent to late HMR findings (4 hours post injection). When compared with FP-CIT imaging (3-6 hours post injection), early MIBG could represent a more acceptable investigation. Planar MIBG data can also be acquired over ten minutes, with the patient in a seated or semi-recumbent position, while FP-CIT requires supine positioning over a 20-minute acquisition period. MIBG is cheaper than FP-CIT and confers a lower dose of radiation, factors which may encourage MIBG in preference to FP-CIT use in selected patients.

A limitation of our study is our modest sample size, and caution should be exercised in dismissing the effect of interacting medications and comorbidities on the basis of our results alone. Our use of clinical diagnosis as the gold standard, rather than neuropathological diagnosis, limits the strength of our findings, although this method has been validated 
against autopsy data[30]. This is perhaps underlined by the presence of two cases meeting clinical criteria for DLB both of whom had normal MIBG or FP-CIT scans.

Our findings could have been further supported by the use of semiquantitative FP-CIT analysis and the use of reference HMR data for our clinical population. The former was not included in our study as we wished our methodology to reflect current routine clinical practice, which favours visual analysis; the latter was not used due to a lack of availability of such data. It would also have been strengthened by the collection of cardiac neuropathological data, as a normal HMR does not exclude the possibility of cardiac Lewy body deposition[28]. Further research into the relationship between HMR and $\alpha$-synuclein pathology is needed.

\section{CONCLUSIONS}

MIBG demonstrates moderate sensitivity and specificity for distinguishing probable DLB from probable $A D$ in a clinically representative population, and has some potential advantages over FP-CIT. Determination of a suitable HMR cut-off is important for optimising clinical accuracy, especially for enhancing specificity, and may be lower in a Caucasian population than those observed in Japanese populations. Our finding that neither medication, nor previous $\mathrm{MI}$, had a significant effect on MIBG uptake ought to encourage further research into this biomarker using larger clinically representative samples. 


\section{DECLARATIONS}

\section{Ethics approval and consent to participate}

Ethical approval for the study was awarded by an NHS Regional Ethics Committee (NRES Committee North East - Newcastle \& North Tyneside 1; Reference 15/NE/0034).

\section{Consent for publication}

Not applicable.

\section{Availability of data and material}

The datasets used and/or analysed during the current study are available from the corresponding author on reasonable request.

\section{Competing interests}

The authors have no competing interests to declare.

\section{Funding}

The study was supported provided by the Newcastle National Institute for Health Research (NIHR) Biomedical Research Centre, hosted by Newcastle upon Tyne Hospitals NHS Foundation Trust and Newcastle University.

\section{Authors' contributions}

JK was responsible for the day to day management of the study, under the supervision of AT, and lead data analysis and the writing of this manuscript. JK, JJ, JOB and AT were responsible for the development of the study protocol. GR and JK were responsible for 
MIBG analysis and all of the authors responsible for FP-CIT analysis. Each of the authors read, contributed to, and approved the final manuscript.

\section{Acknowledgements}

The authors would like to thank the support provided by the Newcastle National Institute for Health Research (NIHR) Biomedical Research Centre, hosted by Newcastle upon Tyne Hospitals NHS Foundation Trust and Newcastle University, and in particular, the work of Miss Helen Kain, Research Support Secretary. Professor John O’Brien is supported by the Cambridge NIHR Biomedical Research Centre.

We thank our colleagues at the Dementia and Neurodegenerative Diseases Research Network, the Medical Physics team at the Newcastle Royal Victoria Infirmary, the clinicians throughout the North-East who assisted in the study, Join Dementia Research, and the study sponsors, Newcastle upon Tyne Hospitals NHS Foundation Trust. 


\section{REFERENCES}

1 McKeith IG, Boeve BF, Dickson DW, et al. Diagnosis and management of dementia with Lewy bodies. Neurology 2017;89:88-100.

2 Vann Jones SA, O'Brien JT. The prevalence and incidence of dementia with Lewy bodies: a systematic review of population and clinical studies. Psychol Med 2014;44:673-83.

3 Kane JPM, Surendranathan A, Bentley A, et al. Clinical prevalence of Lewy body dementia. Alzheimer's Res Ther 2018;10:1-8.

4 Lee DR, McKeith IG, Mosimann U, et al. Examining carer stress in dementia: the role of subtype diagnosis and neuropsychiatric symptoms. Int J Geriatr Psychiatry 2013;28:135-41.

5 Galvin JE, Duda JE, Kaufer DI, et al. Lewy body dementia: the caregiver experience of clinical care. Parkinsonism Relat Disord 2010;16:388-92.

6 Beach TG, Adler CH, Sue LI, et al. Multi-organ distribution of phosphorylated alphasynuclein histopathology in subjects with Lewy body disorders. Acta Neuropathol 2010;119:689-702.

7 Yoshita M, Taki J, Yamada M. A clinical role for [ ${ }^{123}$ I] MIBG myocardial scintigraphy in the distinction between dementia of the Alzheimer's-type and dementia with Lewy bodies. J Neurol Neurosurg Psychiatry 2001;71:583-8.

8 Noguchi-Shinohara M, Tokuda T, Yoshita M, et al. CSF alpha-synuclein levels in dementia with Lewy bodies and Alzheimer's disease. Brain Res 2009;1251:1-6.

9 Shimizu S, Hirao K, Kanetaka H, et al. Utility of the combination of DAT SPECT and MIBG myocardial scintigraphy in differentiating dementia with Lewy bodies from Alzheimer's disease. Eur J Nucl Med Mol Imaging 2016;43: 184-192. 
10 Yoshita $\mathrm{M}$, Arai $\mathrm{H}$, Arai $\mathrm{H}$, et al. Diagnostic accuracy of ${ }^{123}$-meta-iodobenzylguanidine myocardial scintigraphy in dementia with Lewy bodies: a multicenter study. PLoS One 2015;10:e0120540.

11 Komatsu, J, Samuraki, M, Nakajima et al. ${ }^{123}$ I-MIBG myocardial scintigraphy for the diagnosis of DLB: a multicentre 3-year follow-up study. J Neurol Neurosurg Psychiatry Published Online First: 31 May 2018. doi: 10.1136/jnnp-2017-317398

12 Estorch $\mathrm{M}$, Camacho V, Paredes $\mathrm{P}$, et al. Cardiac ${ }^{123}$ I-metaiodobenzylguanidine imaging allows early identification of dementia with Lewy bodies during life. Eur J Nucl Med Mol Imaging 2008;35:1636-41.

13 Novellino F, Bagnato A, Salsone M, et al. Myocardial ${ }^{123}$ I-MIBG scintigraphy for differentiation of Lewy bodies disease from FTD. Neurobiol Aging 2010;31:1903-11.

14 Finegold JA, Asaria P, Francis DP. Mortality from ischaemic heart disease by country, region, and age: Statistics from World Health Organisation and United Nations. Int J Cardiol 2013;168:934-45.

15 Giammarile $\mathrm{F}$, Chiti $\mathrm{A}$, Lassmann $\mathrm{M}$, et al. EANM procedure guidelines for ${ }^{131}$ I-metaiodobenzylguanidine ( ${ }^{131} \mathrm{I}-\mathrm{mIBG}$ ) therapy. Eur J Nucl Med Mol Imaging 2008;35:103947.

16 Tiraboschi P, Corso A, Guerra UP, et al. ${ }^{123}$ I-2 $\beta$-carbomethoxy-3 $\beta$-(4-iodophenyl)-N-(3Fluoropropyl) nortropane single photon emission computed tomography and ${ }^{123}$ metaiodobenzylguanidine myocardial scintigraphy in differentiating dementia with Lewy bodies from other dementias: A comparative study. Ann Neurol 2016;80:36878.

17 Jacobson AF, Travin MI. Impact of medications on mIBG uptake, with specific attention to the heart: Comprehensive review of the literature. J Nucl Cardiol 2015;22:980-93. 
Thomas AJ, Attems J, Colloby SJ, et al. Autopsy validation of ${ }^{123} \mathrm{I}-\mathrm{FP}-\mathrm{CIT}$ dopaminergic neuroimaging for the diagnosis of DLB. Neurology 2017;88:276-83.

19 McKeith IG, O'Brien JT, Walker Z, et al. Sensitivity and specificity of dopamine transporter imaging with ${ }^{123}$ I-FP-CIT SPECT in dementia with Lewy bodies: a phase III, multicentre study. Lancet Neurol 2007;6:305-13.

20 Morgan S, Kemp P, Booij J, et al. Differentiation of frontotemporal dementia from dementia with Lewy bodies using FP-CIT SPECT. J Neurol Neurosurg Psychiatry 2012;83:1063-70.

21 Zijlmans J, Evans A, Fontes F, et al. [ $\left.{ }^{123} \mathrm{I}\right] \mathrm{FP}-\mathrm{CIT}$ spect study in vascular parkinsonism and Parkinson's disease. Mov Disord 2007;22:1278-85.

22 Matsunari I, Schricke U, Bengel FM, et al. Extent of cardiac sympathetic neuronal damage is determined by the area of ischemia in patients with acute coronary syndromes. Circulation 2000;101:2579-85.

23 McKhann GM, Knopman DS, Chertkow $\mathrm{H}$, et al. The diagnosis of dementia due to Alzheimer's disease: Recommendations from the National Institute on AgingAlzheimer's Association workgroups on diagnostic guidelines for Alzheimer's disease. Alzheimer's Dement 2011;7:263-9.

24 Nakajima K, Verschure DO, Okuda K, et al. Standardization of ${ }^{123} \mathrm{I}-$ meta- $^{-}$ iodobenzylguanidine myocardial sympathetic activity imaging: phantom calibration and clinical applications. Clin Transl Imaging 2017;5:255-63. Benamer HTS, Patterson J, Grosset DG, et al. Accurate differentiation of parkinsonism and essential tremor using visual assessment of [ $\left.{ }^{123} \mathrm{I}\right]-\mathrm{FP}-\mathrm{CIT}$ SPECT imaging: The $\left[{ }^{123} \mathrm{I}\right]-$ FP-CIT study group. Mov Disord 2000;15:503-10. 
26 Chen, W, Cao, Q, Dilsizian, V. Variation of Heart-to-Mediastinal Ratio in 123I-mIBG Cardiac Sympathetic Imaging: Its Affecting Factors and Potential Corrections. Curr. Cardiol. Rep. 2011;13:132-137.

27 Bhatnagar $\mathrm{P}$, Wickramasinghe $\mathrm{K}$, Williams J, et al. The epidemiology of cardiovascular disease in the UK 2014. Heart 2015;101:1182-9.

28 Surmeier DJ, Obeso JA, Halliday GM. Selective neuronal vulnerability in Parkinson disease. Nat Rev Neurosci 2017;18:101-13.

29 Takahashi M, Ikemura M, Oka T, et al. Quantitative correlation between cardiac MIBG uptake and remaining axons in the cardiac sympathetic nerve in Lewy body disease. $J$ Neurol Neurosurg Psychiatry 2015;86:939-44.

30 McKeith IG, Ballard CG, Perry RH, et al. Prospective validation of consensus criteria for the diagnosis of dementia with Lewy bodies 207. Neurology 2000;54:1050-8. 


\section{LEGENDS}

Table 1. Demographic and clinical characteristics of probable DLB and probable AD groups

Table 2. Patients prescribed potentially interacting medications

* $\quad$ False positive result

** $\quad$ False negative result

$+\quad$ Theoretical interaction [20]

$+\quad$ Probable interaction [20]

Figure 1. Late anterior planar images of MIBG uptake in two study participants. Both depict freehand heart ROIs $(H)$ and 48-pixel mediastinal ROIs (M). Picture a demonstrates normal cardiac MIBG update (HMR 3.27); b demonstrates decreased uptake (HMR 1.46) indicative of an abnormal scan result.

Figure 2. MIBG (Late HMR) and FP-CIT in probable DLB and probable AD 


\section{FIGURES}

Figure 1. Late anterior planar images of MIBG uptake in two study participants. Both depict freehand heart ROIs $(H)$ and 48-pixel mediastinal ROIs (M). Picture a demonstrates normal cardiac MIBG update (HMR 3.27); b demonstrates decreased uptake (HMR 1.46) indicative of an abnormal scan result.

Figure 2. MIBG (Late HMR) and FP-CIT in probable DLB and probable AD 